\title{
MANAGING UNINTENTIONAL IATROGENIC TRIPLE LUMEN CENTRAL VENOUS CATHETER (TLCVC) ARTERIAL PUNCTURE
}

\author{
Ezamin Abdul Rahim ${ }^{1 *}$, Nurulfida' Nusaiba M. Shukor ${ }^{1}$ Ahmad Sobri Muda ${ }^{1}$, Hariati \\ Jamil $^{2}$, Heamn N. Abduljabbar ${ }^{3}$, Ridzuan Abdul Rahim ${ }^{4}$ \\ ${ }^{1}$ Department of Imaging, Faculty of Medicine and Health Sciences, Universiti Putra Malaysia, Selangor, Malaysia \\ ${ }^{2}$ Putrajaya Hospital, Precinct 7, Putrajaya, Malaysia \\ ${ }^{3}$ Salahaddin University, College of Education, Shaqlawa, Iraq \\ ${ }^{4}$ National Cancer Institute, Precinct 7, Putrajaya, Malaysia
}

\section{*Corresponding author:}

Dr. Ezamin Abdul Rahim, Department of Imaging, Faculty of Medicine and Health Sciences, Universiti Putra Malaysia, 43600, Serdang, Selangor, Malaysia. Email: drezahar@gmail.com

DOI: https://doi.org/10.32896/tij.v1n2.12-18

Published: 31.12 .2021

\begin{abstract}
Unintentional arterial puncture by central venous catheter insertion/placing can result in destructive complications especially if a large bore $(>7 \mathrm{~F})$ catheter was used. Unplanned immediate catheter ejection with manual external compression is hazardous due to potential torrential blood leakage or formation of a pseudoaneurysm. Endovascular removal with a vascular closure device deployment is preferred in this situation. The aim of this case report is to discuss the alternative strategies if the vascular closure device fails to secure hemostasis.
\end{abstract}

Keywords: Angiography, Arterial cannulation, Angioseal.

\section{CASE REPORT}

A 32 years old lady, had an abortion in her second trimester. A post-evacuation of retained products of conception (ERPOC) was commenced; however, it was complicated with postpartum haemorrhage. Subsequently, the patient developed severe pre-eclampsia with acute pulmonary oedema. An 8Fr triple lumen central venous catheter (TLCVC) was inserted under ultrasound guidance in the intensive care unit (ICU).
However, the post-TLCVC insertion chest X-ray showed that the tip of the catheter was not located at the intended location. Instead, it was located at the aortic arch area (Figure 1). A subsequent CT angiography (CTA) showed that the TLCVC had passed through the right internal jugular vein wall piercing into the right subclavian artery. The arterial entry point was identified just immediately distal to the bifurcation of the brachiocephalic trunk (Figure 2). 


\section{IODlle p supine}

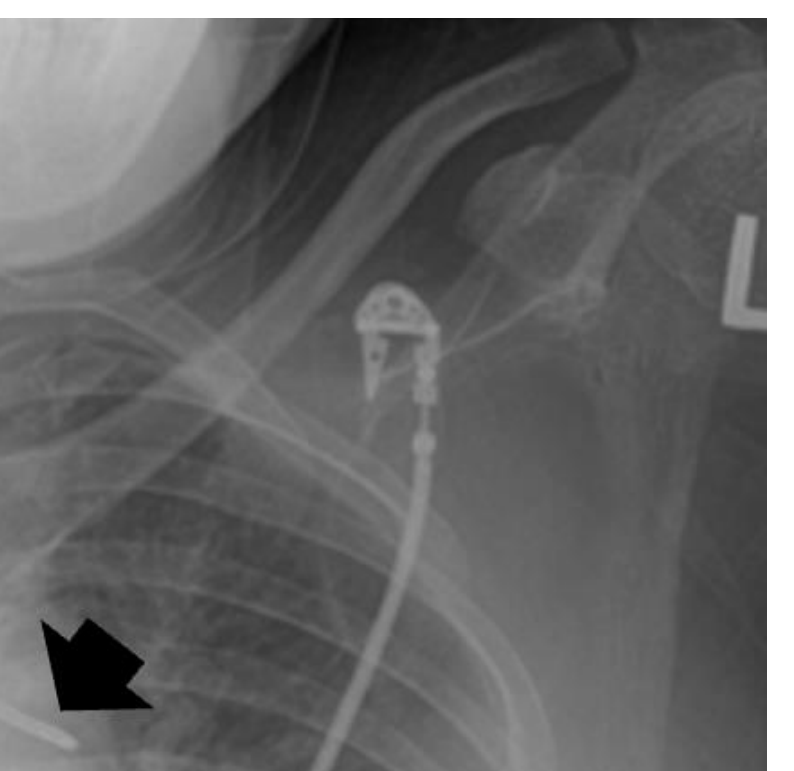

Figure 1: Moderate upper mediastinal haematoma (black long arrow). Tip of the TLCVC is located at the arch of aorta (black arrowhead). 


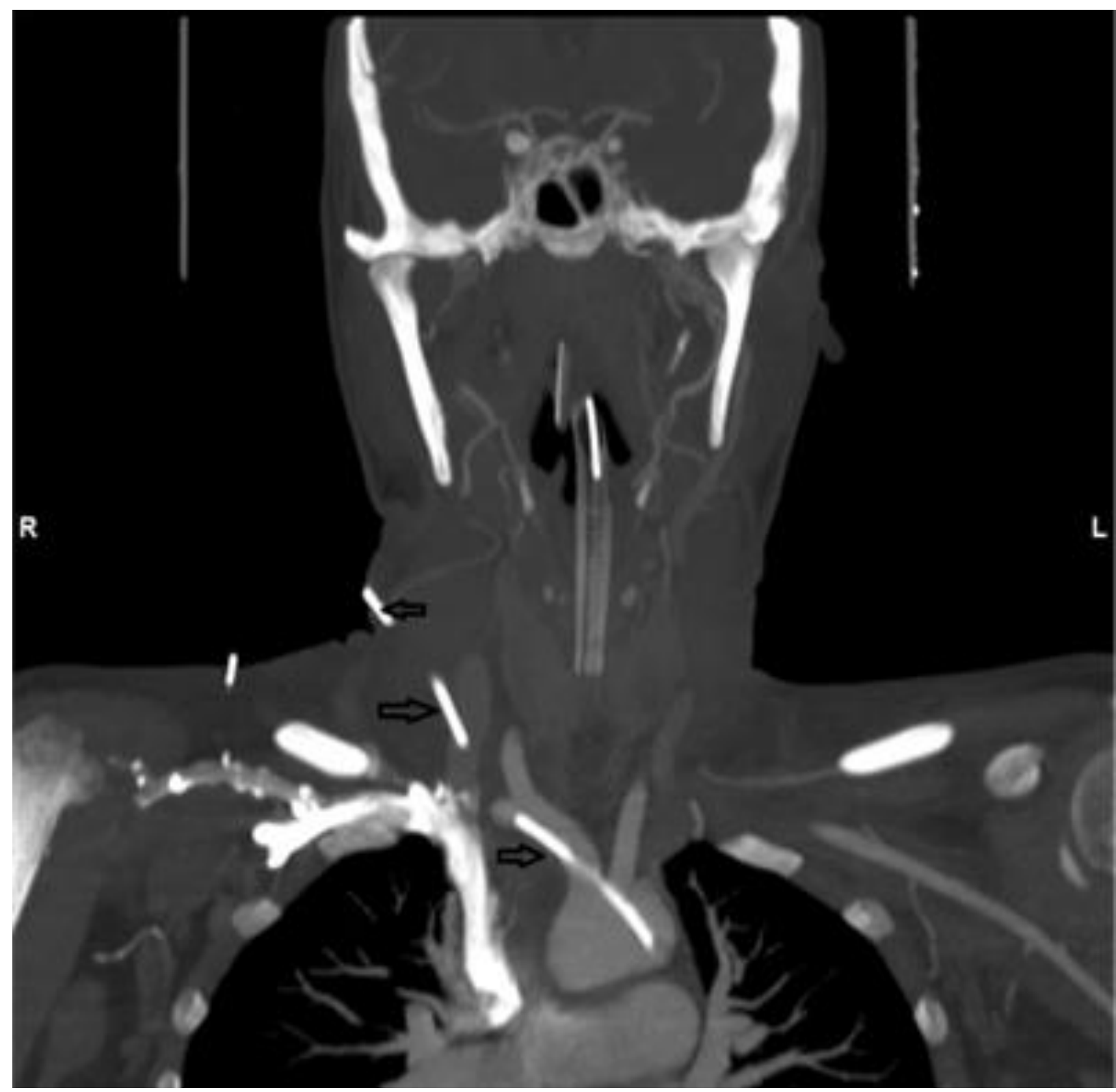

Figure 2: Blank arrows showing the inadvertent TLCVC

The patient remained stable in ICU. The endovascular treatment was considered. The initial plan was to seal the arterial puncture site with a vascular closing device ( $8 \mathrm{Fr}$ angio-seal VIP-Terumo). The procedure was done under local anaesthesia. The patency of right internal cerebral artery and the right vertebral artery were first checked by cerebral angiogram through the femoral sheath (Figure 3). A 6Fr guiding sheath
(Teleflex Arrow) was left at the right brachiocephalic artery. The aim of this guiding sheath is to facilitate balloon inflation. A $260 \mathrm{~cm}$ 0.035 " inch guide wire was also left with its tip at the right axillary artery. This was to ease the catheterization of the endovascular balloon device if the vascular closure device failed. This endovascular balloon will function as a tamponade. 


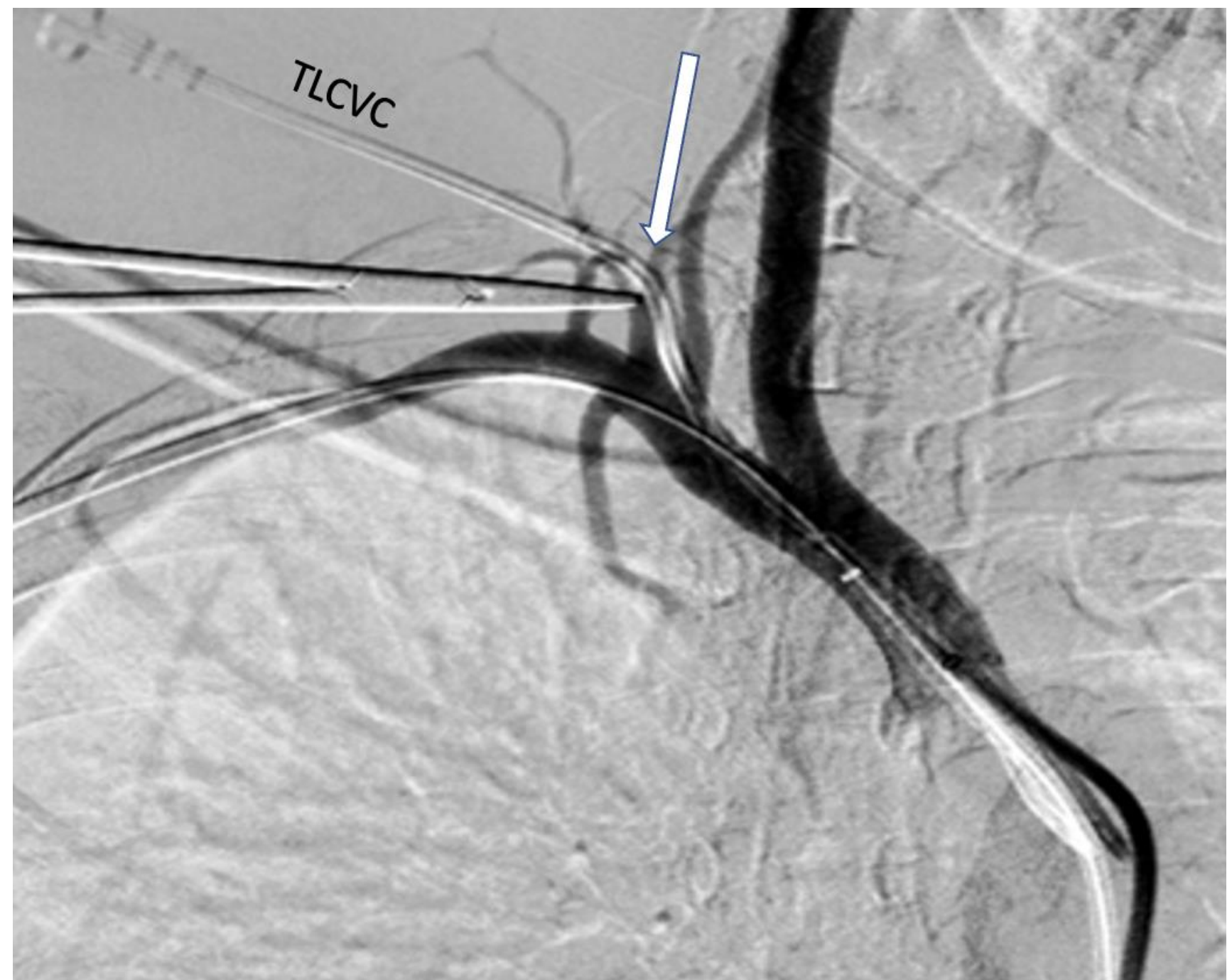

Figure 3: The right subclavian artery, the right vertebral artery and their branches were patent (Angiographic run prior to removal of TLCVC). Note the angulation of TLCVC that caused the failure of the angio-seal VIP device insertion.

\section{Step by step narrative:}

A 0.035 " guide wire was then inserted through the central lumen of the inadvertent TLCVC. The guide wire tip was placed in the arch of the aorta to ensure that there is enough length of the guide wire during exchange with the angio-seal locator system. The TLCVC was then removed. This was done quickly with external compression of the site of puncture. The angio-seal insertion sheath was inserted first; followed by the arteriotomy locator device. These 2 components were successfully placed within the artery, the arteriotomy locator and guide wire were removed whilst holding the angioseal introducer.
The angio-seal VIP device was subsequently inserted into the sheath hub, however the operator was not able to advance the device further to its full length. During the struggle, the operator accidently dislodged the sheath hub and the partially inserted angio-seal VIP assembly from the intended arterial puncture site. The operator immediately did manual local compression to the neck. An angiographic run was done during the chaotic moment through the $6 \mathrm{Fr}$ guiding sheath that showed a massive contrast leakage from the TLCVC arterial puncture site at the right subclavian artery (Figure 4). 


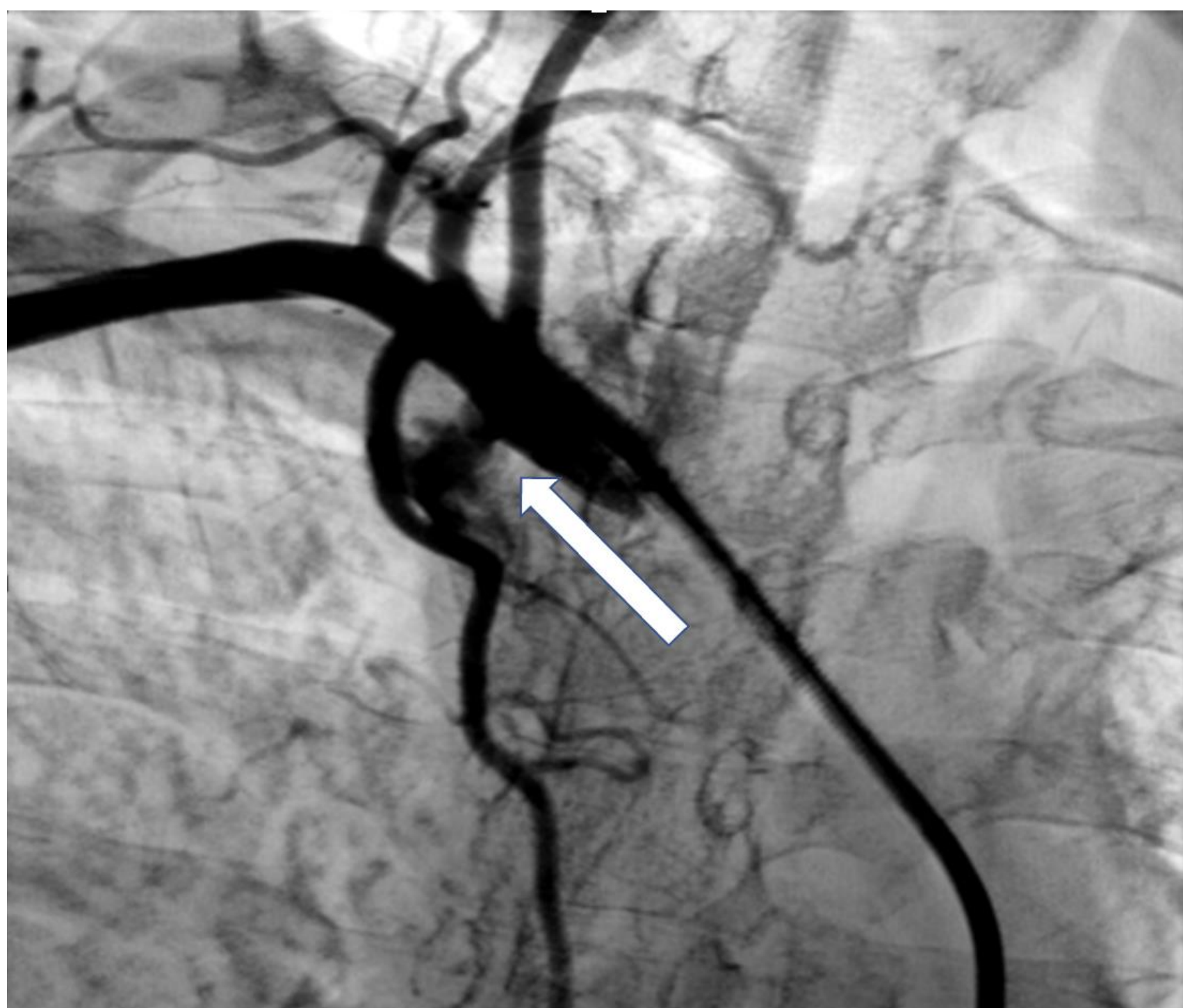

Figure 4: Angiographic run showed massive contrast leakage from the puncture site at the right subclavian artery (white arrow).

A balloon sized $8 \mathrm{~mm} \times 40 \mathrm{~mm}$ was immediately inserted and inflated at the site of the proximal right subclavian artery (arterial puncture site). The balloon acted as a tamponade to reduce extravasation thus promoting haemostasis (Figure
5). Inflation and deflation of the balloon at the intended area and angiographic run were done several times. The last angiographic run showed there was no more contrast leakage seen from the inadvertent arterial TLCVC puncture site. 


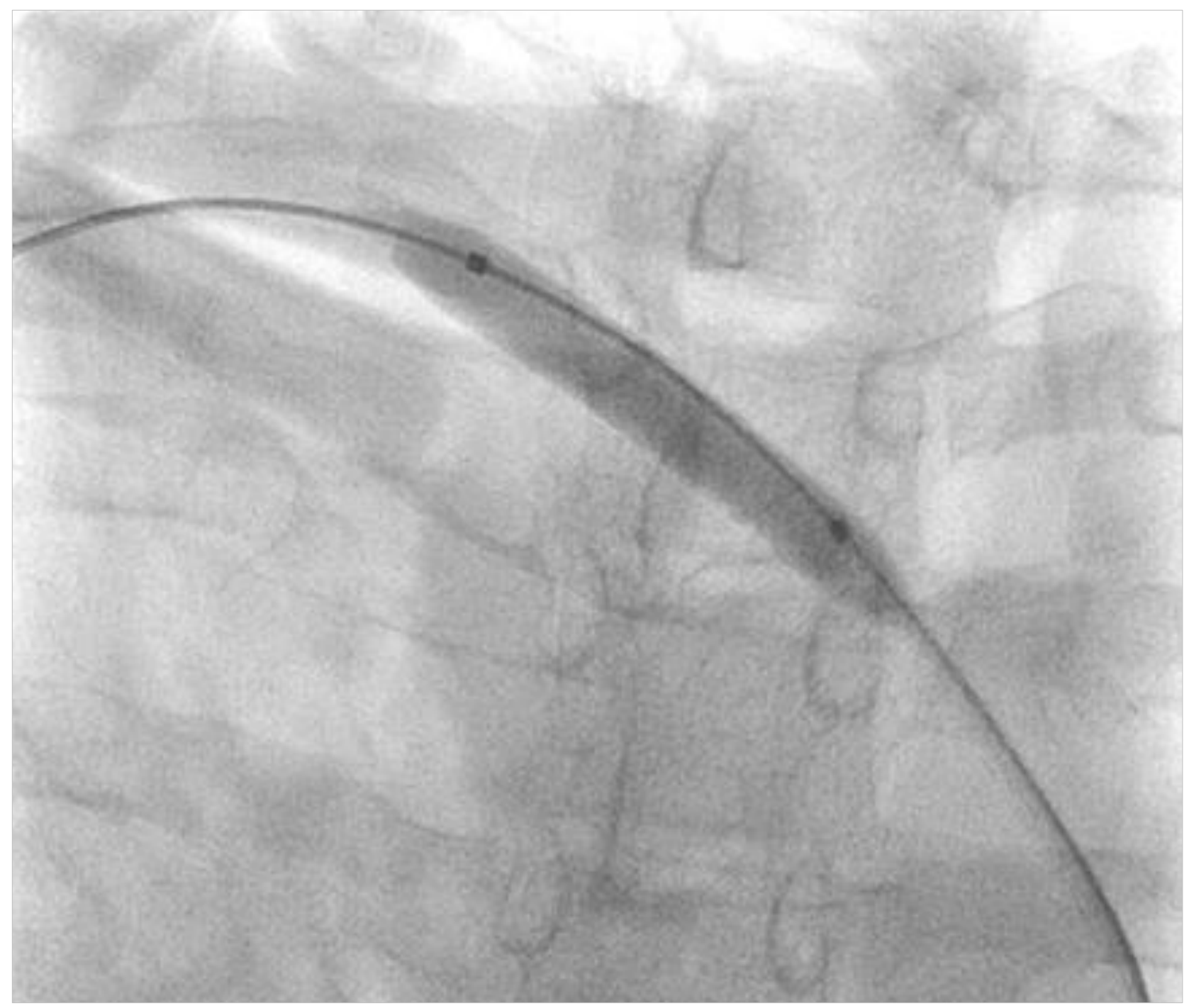

Figure 5: shows endovascular balloon inflation at the site of leakage.

The femoral sheath was left in situ and secured with Tegaderm in preparation of a re-bleeding event. The patient was stable throughout the procedure. Patient only complained of pain in the right neck region during the manual compression. She was closely monitored in the intensive care unit and discharged to the general ward after 2 days.

\section{DISCUSSION}

Catheter-related cervicothoracic arterial injury is a known complication related to central venous catheter insertion. However, currently there are no definite guidelines on handling accidental largebore arterial cannulation. Generally, the management may include removal and manual external compression, endovascular intervention or surgical exploration. Simply removing the inadvertent TLCVC followed by immediate local manual compression technique has a relatively high morbidity rate. The complications that may occur with this simple technique are airway obstruction due to haematoma, stroke and pseudoaneurysm. Surgery is the gold standard with a higher success rate; however, exploratory neck surgery is an invasive procedure.

Endovascular arterial plug serves as an alternative approach in such cases. Using the advantage of the mechanical seal of copolymer anchor and collagen sponge to close the accidental 
arteriotomy site. The seal is held securely in place by a self-tightening suture. All these mechanical parts will be absorbed within 90 days. The Angioseal device is meant to be used as an arterial closure at the femoral artery. The usage of this device on other than the femoral artery is termed as off-label use.

The failure of this device in our case is probably due to acute angulation of the inadvertent puncture tract. This acute angulation caused the angioseal introducer sheath to bend thus preventing the angioseal device to be locked in the designated position as shown in Figure 3.
It was reported that arterial closure device failed in $4.9 \%$ of cases. Minor complications such as recurrent wound bleeding or hematoma were also reported to be $3.8 \%$. $(1,2,3)$. Variables such as obesity, emergency procedure, and short neck pose higher risk for failure $(4,5,6,7)$.

\section{CONCLUSION}

This case report is done to highlight a simple algorithm in managing iatrogenic triple lumen central venous catheter (TLCVC) arterial puncture, in which intra-arterial balloon tamponade and external compression are applied simultaneously.

\section{REFERENCES}

1. Pikwer A, Acosta S, Kölbel T, Malina M, Sonesson B, Åkeson J. Management of inadvertent arterial catheterisation associated with central venous access procedures. European journal of vascular and endovascular surgery. 2009 Dec 1;38(6):707-14.

https://www.sciencedirect.com/science/article/pii/S1078588409004419

2. Guilbert MC, Elkouri S, Bracco D, Corriveau MM, Beaudoin N, Dubois MJ, Bruneau L, Blair JF. Arterial trauma during central venous catheter insertion: Case series, review and proposed algorithm. Journal of vascular surgery. 2008 Oct 1;48(4):918-25.

https://pubmed.ncbi.nlm.nih.gov/18703308/

3. Bowdle A. Vascular complications of central venous catheter placement: evidence-based methods for prevention and treatment. Journal of cardiothoracic and vascular anesthesia. 2014 Apr 1;28(2):358-68. https://pubmed.ncbi.nlm.nih.gov/24008166/

4. Wójtowicz D, Cholewa D, Faba AM, Domańska B, Kokoszka J, Kopacz K, Ficek R, Irzyniec T, Rotkegel SE, Chudek J. Diabetes decreases patency of tunneled catheters in hemodialysis patients after first effective thrombolysis with urokinase. Renal failure. 2018 Oct 15;40(1):384-9. https://www.ncbi.nlm.nih.gov/pmc/articles/PMC6052429/

5. Paliwal B, Rai P, Kamal M, Singariya G, Singhal M, Gupta P, Trivedi T, Chouhan DS. Comparison between dexmedetomidine and propofol with validation of bispectral index for sedation in mechanically ventilated intensive care patients. Journal of clinical and diagnostic research: JCDR. 2015 Jul;9(7):UC01. https://www.ncbi.nlm.nih.gov/pmc/articles/PMC4573016/

6. Lucatelli P, Fanelli F, Cannavale A, Corona M, Cirelli C, D'Adamo A, Salvatori FM, Catalano C. Angioseal VIP® vs. StarClose SE® closure devices: a comparative analysis in non-cardiological procedures. The Journal of cardiovascular surgery. 2017 Feb;58(1):80-6.

https://www.minervamedica.it/en/journals/cardiovascularsurgery/article.php?cod=R37Y2017N01A0080

7. Liu F, Hansra S, Crockford G, Köster W, Allan BJ, Blondeau JM, Lainesse C, White AP. Tetrasodium EDTA Is Effective at Eradicating Biofilms Formed by Clinically Relevant Microorganisms from Patients' Central Venous Catheters. mSphere. 2018 Dec 26;3(6):e0052518. https://pubmed.ncbi.nlm.nih.gov/30487154/ 